\title{
ON AN IMPROVEMENT OF A THEOREM OF T. NAGELL CONCERNING THE DIOPHANTINE EQUATION
}$$
A x^{3}+B y^{3}=C
$$ \\ WILHELM LJUNGGREN
}

1. The Diophantine equation

$$
x^{3}+D y^{3}=1,
$$

where $D$ denotes a positive rational integer, which is not a cube, was solved completely by B. Delaunay [1] who showed that it has at most one solution in rational integers $x$ and $y$ when $y \neq 0$. If $x=x_{1}, y=y_{1}$ is an integral solution, then

$$
\zeta=x_{1}+y_{1} D^{\frac{1}{3}}
$$

is the fundamental unit of the ring $R\left(1, D^{\frac{1}{3}}, D^{\frac{2}{3}}\right)$.

T. Nagell $[5 ; 6 ; 7 ; 8]$ proved the same theorem independently of Delaunay and, moreover, a stronger form of the latter part of the theorem.

Nagell $[7 ; 8]$ proved that $\zeta$ is the fundamental unit of the field $K\left(D^{\frac{1}{1}}\right)$, except when $D=19,20$ and 28 , in which cases $\zeta$ is the square of the fundamental unit. These values of $D$ correspond to the solutions $x=-8, y=3 ; \quad x=-19, y=7 ; \quad$ and $x=-3, y=1$.

Nagell [7] generalized these results, showing that the Diophantine equation

$$
A x^{3}+B y^{3}=C \quad(C=1 \text { or } C=3),
$$

where $A$ and $B$ are $>1$ when $C=1$ and where $A B$ is not divisible by 3 when $C=3$, has at most one solution in rational integers $x$ and $y$.

He also obtained the following result: Put $A=a c^{2}$ and $B=b d^{2}$, where $a, b, c$ and $d$ are positive rational integers, relatively prime in pairs, and possessing no squared factors. If $x=x_{1}, y=y_{1}$ is a solution, then

$$
\zeta=C^{-1}\left(x_{1} A^{\frac{1}{3}}+y_{1} B^{\frac{1}{3}}\right)^{3}=\xi^{2^{r}},
$$

where $\xi$ is the fundamental unit of the field $K\left(\left(a c^{2} b^{2} d\right)^{\frac{1}{3}}\right), 0<\xi<1$, and where $r$ is a rational integer $\geqq 0$.

Received October 18, 1953. 
There is one exception to this theorem, viz. the equation $2 x^{3}+y^{3}=3$, which has the two solutions $x=y=1$ and $x=4, y=-5$. This exception is not taken into consideration in the following.

Without knowing an upper limit for the integer $r$, Nagell succeeded in constructing an algorithm to decide if (2) is solvable or not. In the former case, this algorithm gives a method to determine the solution of the equation (cf. [7, pp. 257 and 263]). This method, a sort of descente finie, is, however, too cumbersome to be practical.

Nagell $[7 ; 9 ; 10]$ has treated the question of determining an upper limit of $r$. His investigations have been continued by P. Hæggmark [2]. Several interesting results are obtained, but no complete solution of this problem has hitherto been found. In this paper we prove that $r \leqq 1$. This is the best possible result, since Nagell [7, pp. 258 and 264] has proved that $r=1$ for an infinity of fields $K\left(\left(a c^{2} b^{2} d\right)^{\frac{1}{3}}\right)$. This yields the following result:

Theorem: The Diophantine equation

$$
A x^{3}+B y^{3}=C,
$$

where $C=1$ or $C=3$, where $A$ and $B$ are $>1$ when $C=1$, and where $A B$ is not divisible by 3 when $C=3$, has at most one solution in rational integers $x$ and $y$. If $x=x_{1}, y=y_{1}$ is an integral solution, then $C^{-1}\left(x_{1} A^{\frac{1}{3}}+y_{1} B^{\frac{1}{3}}\right)^{3}$ is either the fundamental unit in the field $K\left(\left(A B^{2}\right)^{\frac{1}{3}}\right)$ or the square of this unit.

2. Let $\eta, 0<\eta<1$, be a unit in $K\left(\left(A B^{2}\right)^{\frac{1}{3}}\right)$. Then we must have, if $r>1$,

where

$$
C^{-1}\left(x A^{\frac{1}{3}}+y B^{\frac{1}{3}}\right)^{3}=\eta^{4},
$$

$$
\eta^{3}-p \eta^{2}+q \eta-1=0,
$$

$p$ and $q$ denoting rational integers. This gives us

$$
\begin{aligned}
\eta^{4} & =1+3 C^{-1} x^{2} y\left(A^{2} B\right)^{\frac{1}{3}}+3 C^{-1} x y^{2}\left(A B^{2}\right)^{\frac{1}{3}} \\
\eta^{\prime 4} & =1+3 C^{-1} x^{2} y \varrho\left(A^{2} B\right)^{\frac{1}{3}}+3 C^{-1} x y^{2} \varrho^{2}\left(A B^{2}\right)^{\frac{1}{3}}, \\
\eta^{\prime \prime 4} & =1+3 C^{-1} x^{2} y \varrho^{2}\left(A^{2} B\right)^{\frac{1}{3}}+3 C^{-1} x y^{2} \varrho\left(A B^{2}\right)^{\frac{1}{3}}, \quad \varrho^{3}=1, \varrho \neq 1,
\end{aligned}
$$

where $\eta^{\prime}$ and $\eta^{\prime \prime}$ are the conjugates of $\eta$. The equations (5) imply

or

$$
\begin{gathered}
\eta^{4}+\eta^{\prime 4}+\eta^{\prime \prime 4}=3, \\
\left(p^{2}-2 q\right)^{2}-2\left(q^{2}-2 p\right)=3, \\
q=p^{2} \pm(p-1)\left(\frac{1}{2}\left(p^{2}+2 p+3\right)\right)^{\frac{1}{2}},
\end{gathered}
$$


that is

$$
\begin{gathered}
q=p^{2}+(p-1) M, \\
M^{2}-2\left(\frac{1}{2}(p+1)\right)^{2}=1 .
\end{gathered}
$$

From (5) we further obtain

$$
\begin{aligned}
& \eta^{4}+\varrho \eta^{\prime 4}+\varrho^{2} \eta^{\prime \prime 4}=9 C^{-1} x y^{2}\left(A B^{2}\right)^{\frac{1}{3}}, \\
& \eta^{4}+\varrho^{2} \eta^{\prime 4}+\varrho \eta^{\prime 4}=9 C^{-1} x^{2} y\left(A^{2} B\right)^{\frac{1}{3}} .
\end{aligned}
$$

By multiplication of these two equations we get

$$
\begin{gathered}
\eta^{8}+\eta^{\prime 8}+\eta^{\prime \prime}-\left(\eta \eta^{\prime \prime}\right)^{4}-\left(\eta \eta^{\prime}\right)^{4}-\left(\eta^{\prime} \eta^{\prime \prime}\right)^{4}=81 C^{-2} A B x^{3} y^{3}, \\
\left(\eta^{4}+\eta^{\prime 4}+\eta^{\prime \prime 4}\right)^{2}-3\left(\left(\eta \eta^{\prime \prime}\right)^{4}+\left(\eta \eta^{\prime}\right)^{4}+\left(\eta^{\prime} \eta^{\prime \prime}\right)^{4}\right)=81 C^{-2} A B x^{3} y^{3}, \\
9-3\left(\left(q^{2}-2 p\right)^{2}-2\left(p^{2}-2 q\right)\right)=81 C^{-2} A B x^{3} y^{3} .
\end{gathered}
$$

From (7) we find $q^{2}-2 p=\frac{1}{2}\left(\left(p^{2}-2 q\right)^{2}-3\right)$; inserting this expression in (10) we get the result

$$
3-\left(p^{2}-2 q\right)^{4}+6\left(p^{2}-2 q\right)^{2}+8\left(p^{2}-2 q\right)=108 C^{-2} A B x^{3} y^{3} .
$$

Putting for brevity $p^{2}-2 q=t$, this equation can be written

$$
(t+1)^{3}(t-3)=-108 C^{-2} A x^{3}\left(C-A x^{3}\right) ;
$$

hence

that is,

$$
6 C^{-1} A x^{3}=3+(t-2)\left(\frac{1}{3}\left(t^{2}+4 t+6\right)\right)^{\frac{1}{2}},
$$

$$
t^{2}+4 t+6=3 N^{2}
$$

or

$$
\begin{gathered}
\left(p^{2}-2 q+2\right)^{2}+2=3 N^{2}, \\
6 C^{-1} A x^{3}=3+\left(p^{2}-2 q-2\right) N .
\end{gathered}
$$

Consequently, we have to solve the system (9) and (12). This can also be written in the following form

$$
\begin{gathered}
M^{2}-2\left(\frac{1}{2}(p+1)\right)^{2}=1, \\
\left(p^{2}+2(p-1) M-2\right)^{2}+2=3 N^{2},
\end{gathered}
$$

making use of (8). The corresponding values of $q, A, B$ and $C$ are determined by (8) and (13).

In the following sections it will be shown that the only solutions of the system (14) and (15) in rational integers $p, M$ and $N$ are

$$
\begin{gathered}
p=-1, M=1, N= \pm 3 ; \quad p=3, M=-3, N= \pm 3 ; \\
p=3, M=3, N= \pm 11
\end{gathered}
$$


with $q$ equal to $-1,3$ and 15 , respectively. In the first two cases we find either $A x^{3}=C$ or $B y^{3}=C$, which is impossible. In the last case we get the equation $2 x^{3}+y^{3}=3$ with $\eta=\left(1-2^{\frac{1}{3}}\right)^{2}$ and

$$
\frac{1}{3}\left(4 \cdot 2^{\frac{1}{3}}-5\right)^{3}=\left(1-2^{\frac{1}{3}}\right)^{8} .
$$

Then our theorem is proved.

3. We have

$$
\left(p^{2}+2(p-1) M-2\right)^{2}+2=(p+2 M+2)^{2}\left((M-2)^{2}+2\left(\frac{1}{2}(p-1)\right)^{2}\right),
$$

because the value of each side of (16) is found to be equal to

$$
4\left(p^{3}-p^{2}-2 p+2\right) M+3 p^{4}-4 p^{2}-8 p+12,
$$

using that $2 M^{2}=p^{2}+2 p+3$. Instead of solving (14) and (15) we can solve the system

$$
(M-2)^{2}+2\left(\frac{1}{2}(p-1)\right)^{2}=3 N_{1}^{2}, \quad N=N_{1}(p+2 M+2) .
$$

From (18) we deduce

Hence

$$
\begin{gathered}
M-2+\frac{1}{2}(p-1)(-2)^{\frac{1}{2}}=e\left(1+e_{1}(-2)^{\frac{1}{2}}\right)\left(u+v(-2)^{\frac{1}{2}}\right)^{2}, \\
e= \pm 1, e_{1}= \pm 1 .
\end{gathered}
$$

$$
M=2+e\left(u^{2}-2 v^{2}\right)-4 e e_{1} u v, \quad \frac{1}{2}(p+1)=1+e e_{1}\left(u^{2}-2 v^{2}\right)+2 e u v .
$$

Inserting these values in (17) we obtain

or

$$
\left(2+e\left(u^{2}-2 v^{2}\right)-4 e e_{1} u v\right)^{2}-2\left(1+e e_{1}\left(u^{2}-2 v^{2}\right)+2 e u v\right)^{2}=1,
$$

$$
\begin{aligned}
\left(u^{2}-2 v^{2}+8 e_{1} u v\right)^{2}-2(6 u v-e)^{2}-4 e\left(1-e_{1}\right)\left(u^{2}-2 v^{2}\right) & \\
& +16 e\left(e_{1}-1\right) u v=-1 .
\end{aligned}
$$

In this equation we have $e_{1}=1$, because if we had $e_{1}=-1$ we would get

$$
\left(u^{2}+u v+v^{2}\right)^{2}+e\left(u^{2}+u v+v^{2}\right) \equiv 1 \quad(\bmod 3),
$$

that is, $e \equiv 0(\bmod 3)$, which is impossible. Then $(20)$ reduces to

or

$$
\left(u^{2}-2 v^{2}+8 u v\right)^{2}-2(6 u v-e)^{2}=-1,
$$

$$
f(u, v) \equiv u^{4}+16 u^{3} v-12 u^{2} v^{2}-32 u v^{3}+4 v^{4}+24 e u v-1=0 .
$$

According to a theorem of C. L. Siegel [11] the equation $f(u, v)=0$ has only a finite number of solutions in integers $u$ and $v$, because the alge- 
braic curve $f(u, v)=0$ is of genus 3 , but the proof gives no method for determining the possible solutions $u$ and $v$. In the following sections we will show that there are only the trivial solutions

$$
u= \pm 1, v=0 ; \quad u=1, v=1, e=1 ; \quad u=-1, v=-1, e=1 .
$$

These values of $u$ and $v$ give precisely the solutions of $p$ and $M$ mentioned in the first section.

4. From (17) we deduce

$$
M=\frac{1}{2} e_{2}\left(E^{2 n}+E^{\prime 2 n}\right), \quad \frac{1}{2}(p+1)=\frac{1}{2} e_{2} 2^{\frac{1}{2}}\left(E^{2 n}-E^{\prime 2 n}\right),
$$

where $E=1+2^{\frac{1}{2}}, E^{\prime}=1-2^{\frac{1}{2}}, e_{2}= \pm 1$ and $n=0, \pm 1, \pm 2, \pm 3, \ldots$ Inserting these expressions in (19) we get

$$
\left(\frac{1}{2}(1+i) E^{2 n}+\frac{1}{2}(1+i) E^{\prime 2 n}\right) e_{2}-2-i 2^{\frac{1}{2}}=e\left(1+i 2^{\frac{1}{2}}\right)\left(u+i 2^{\frac{1}{2}} v\right)^{2} .
$$

Now we find

where

This yields

$$
\frac{1}{2}(1+i) E^{2 n}+\frac{1}{2}(1+i) E^{\prime 2 n}=(-1)^{n-1} 2^{\frac{1}{2}}+E \vartheta^{2},
$$

\section{Putting}

$$
E \vartheta^{2}-e e_{2}\left(1+i 2^{\frac{1}{2}}\right)\left(u+i v 2^{\frac{1}{2}}\right)^{2}=2^{\frac{1}{2}}\left((-1)^{n}+e_{2}\left(i+2^{\frac{1}{2}}\right)\right) .
$$

and

$$
\theta=\left(E\left(1+i 2^{\frac{1}{2}}\right)\right)^{\frac{1}{2}}=\left(\frac{1}{2} E\left(3^{\frac{1}{2}}+1\right)\right)^{\frac{1}{2}}+i\left(\frac{1}{2} E\left(3^{\frac{1}{2}}-1\right)\right)^{\frac{1}{2}}
$$

we find

$$
\theta_{1}=\left(E^{\prime}\left(1+i 2^{\frac{1}{2}}\right)\right)^{\frac{1}{2}}=-\left(\frac{1}{2} E^{-1}\left(3^{\frac{1}{2}}-1\right)\right)^{\frac{1}{2}}+i\left(\frac{1}{2} E^{-1}\left(3^{\frac{1}{2}}+1\right)\right)^{\frac{1}{2}}
$$

$$
\theta \theta_{1}=i\left(1+i 2^{\frac{1}{2}}\right) \text { and } \theta_{1}=-i E^{\prime} \theta, \theta=-i E \theta_{1} .
$$

The algebraic number field $K(\theta)$ is of the eighth degree, and $K(\theta)=K\left(\theta_{1}\right)$. If $\xi$ is any number in $K(\theta)$, we denote by $\xi^{\prime}, \xi^{\prime \prime}, \xi^{\prime \prime \prime}$ the conjugates obtained by changing in $\xi$ the sign of $\theta$, the signs of $i$ and of $2^{\frac{1}{2}}$, the signs of $i$ and of $2^{\frac{1}{2}}$ and of $\theta$, respectively. The conjugates of $\theta$, obtained in this way, are $-\theta, \theta_{1}$ and $-\theta_{1}$ and those of $\theta_{1}$ are $-\theta_{1},-\theta$ and $\theta$.

The algebraic number

$$
\alpha=\frac{\left(\vartheta \mathbf{E}^{\frac{1}{2}}+\left(e e_{2}\right)^{\frac{1}{2}}\left(1+i 2^{\frac{1}{2}}\right)^{\frac{1}{2}}\left(u+i v 2^{\frac{1}{2}}\right)\right)^{2}}{2^{\frac{1}{2}}\left((-1)^{n}+e_{2}\left(i+2^{\frac{1}{2}}\right)\right)}
$$

is a unit in $K(\theta)$ with relative norm 1 in the subfield $k\left(i, 2^{\frac{1}{2}}\right)$. In fact, we find

$$
\alpha+\alpha^{\prime}=-2+\vartheta^{2} E\left(e_{2}+i\left((-1)^{n}-e_{2} 2^{\frac{1}{2}}\right)\right) \quad \text { and } \quad \alpha \alpha^{\prime}=1 .
$$


Further we find

$$
\begin{aligned}
\left(\alpha+\alpha^{\prime}+2\right)\left((-1)^{n}+e_{2}\left(i+2^{\frac{1}{2}}\right)\right) & =2^{3 / 2} \vartheta^{2} E, \\
\left(\alpha^{\prime \prime}+\alpha^{\prime \prime \prime}+2\right)\left((-1)^{n}-e_{2}\left(i+2^{\frac{1}{2}}\right)\right) & =-2^{3 / 2} \vartheta^{\prime \prime 2} E^{\prime} .
\end{aligned}
$$

By addition of these two equations we get

$$
\left(\alpha+\alpha^{\prime}+\alpha^{\prime \prime}+\alpha^{\prime \prime \prime}+4\right)(-1)^{n}+e_{2}\left(i+2^{\frac{1}{2}}\right)\left(\alpha+\alpha^{\prime}-\alpha^{\prime \prime}-\alpha^{\prime \prime \prime}\right)=8(-1)^{n},
$$

using the fact that $\vartheta^{2} E-\vartheta^{\prime \prime 2} E^{\prime}=2^{3 / 2}(-1)^{n}$, which follows easily from (23). Consequently:

$$
\left(\alpha+\alpha^{\prime}+\alpha^{\prime \prime}+\alpha^{\prime \prime \prime}\right)+e_{2}(-1)^{n}\left(i+2^{\frac{1}{2}}\right)\left(\alpha+\alpha^{\prime}-\alpha^{\prime \prime}-\alpha^{\prime \prime \prime}\right)=4 .
$$

In the number field $K(\theta)$ there are 3 independent units, and it is easily shown that the group of units with relative norm 1 in the subfield $k\left(i, 2^{\frac{1}{2}}\right)$ is generated by two independent units, say $\varepsilon_{1}$ and $\varepsilon_{2}$ (cf. Ljunggren $[3, \mathrm{p} .8])$. Then we must have

$$
\alpha= \pm \varepsilon_{1}^{x} \varepsilon_{2}^{y},
$$

because \pm 1 are the only roots of unity whose squares equal 1 . Inserting this in (26) we get two exponential equations to determine the exponents $x$ and $y$, and therefore we can make use of the $p$-adic method developed by Th. Skolem in a series of papers $[12 ; 13 ; 14 ; 15]$.

5. In the same way as in my paper [4, pp. 13-17] it can be shown that

$$
\varepsilon_{1}=\frac{\left(E^{\frac{1}{2}}+i\left(1+i 2^{\frac{1}{2}}\right)^{\frac{1}{2}}\right)^{2}}{2^{\frac{1}{2}}(E+i)}=\frac{1}{2}\left(-i-E^{\prime}+\theta\left(i-E^{\prime}\right)\right)
$$

and

$$
\varepsilon_{2}=\frac{\left(E^{\prime \frac{1}{2}}-i\left(1+i 2^{\frac{1}{2}}\right)^{\frac{1}{2}}\right)^{2}}{-2^{\frac{1}{2}}\left(E^{\prime}-i\right)}=\varepsilon_{1}^{\prime \prime}=\frac{1}{2}\left(i-E-\theta\left(i+E^{\prime}\right)\right)
$$

is a pair of fundamental units. Further we note the units

$$
\begin{aligned}
& \varepsilon_{1} \varepsilon_{2}{ }^{-2}=\frac{\left(E^{\frac{1}{2}}\left(-i E^{\prime}+2^{\frac{1}{2}}\right)+\left(1+i 2^{\frac{1}{2}}\right)^{\frac{1}{2}}\right)^{2}}{2^{\frac{1}{2}}(E+i)}, \\
& \varepsilon_{1}{ }^{3}=\frac{\left(E^{\frac{1}{2}}\left(1+i E^{\prime} 2^{\frac{1}{2}}\right)+i\left(1+i 2^{\frac{1}{2}}\right)\left(1+i 2^{\frac{1}{2}}\right)^{\frac{1}{2}}\right)^{2}}{-2^{\frac{1}{2}}(E+i)} .
\end{aligned}
$$

For the sake of brevity we write

$$
\begin{gathered}
s\left(\varepsilon_{1}^{x} \varepsilon_{2}^{y}\right)=\varepsilon_{1}^{x} \varepsilon_{2}^{y}+\varepsilon_{1}{ }^{x} \varepsilon_{2}{ }^{y}+\varepsilon_{1}{ }^{\prime \prime x} \varepsilon_{2}{ }^{\prime \prime y}+\varepsilon_{1}{ }^{\prime \prime \prime x} \varepsilon_{2}{ }^{\prime \prime \prime y}, \\
d\left(\varepsilon_{1}{ }^{x} \varepsilon_{2}{ }^{y}\right)=\varepsilon_{1}{ }^{x} \varepsilon_{2}{ }^{y}+\varepsilon_{1}{ }^{\prime x} \varepsilon_{2}{ }^{y}-\varepsilon_{1}{ }^{\prime \prime x} \varepsilon_{2}{ }^{\prime \prime y}-\varepsilon_{1}{ }^{\prime \prime \prime} \varepsilon_{2}{ }^{\prime \prime \prime y}
\end{gathered}
$$


and $e_{2}(-1)^{n}=t$. Hence, from (26) and (27)

$$
s\left(\varepsilon_{1}^{x} \varepsilon_{2}^{y}\right)+t\left(i+2^{\frac{1}{2}}\right) d\left(\varepsilon_{1}^{x} \varepsilon_{2}^{y}\right)= \pm 4 .
$$

We first prove some lemmas:

LEMMA 1 : If $(x, y)$ is a solution of $(28)$, then $(-x,-y)$ is also a solution.

This follows immediately from the equations $\varepsilon_{1} \varepsilon_{1}{ }^{\prime}=1, \varepsilon_{1}{ }^{\prime \prime} \varepsilon_{1}{ }^{\prime \prime \prime}=1$, $\varepsilon_{2} \varepsilon_{2}{ }^{\prime}=1, \varepsilon_{2}{ }^{\prime \prime} \varepsilon_{2}{ }^{\prime \prime \prime}=1$.

Lemma 2: If $(x, y)$ is a solution of $(28)$, then $(-y, x)$ is a solution of

$$
s\left(\varepsilon_{1}{ }^{x} \varepsilon_{2}^{y}\right)-t\left(i+2^{\frac{1}{2}}\right) d\left(\varepsilon_{1}^{x} \varepsilon_{2}^{y}\right)= \pm 4 .
$$

Since $\varepsilon_{2}=\varepsilon_{1}{ }^{\prime \prime}, \varepsilon_{2}{ }^{\prime}=\varepsilon_{1}{ }^{\prime \prime}, \varepsilon_{2}{ }^{\prime \prime}=\varepsilon_{1}{ }^{\prime}, \varepsilon_{2}{ }^{\prime \prime \prime}=\varepsilon_{1}$, we have $s\left(\varepsilon_{1}{ }^{x} \varepsilon_{2}{ }^{y}\right)=$ $s\left(\varepsilon_{1}^{-y} \varepsilon_{2}^{x}\right)$ and $d\left(\varepsilon_{1}^{x} \varepsilon_{2}^{y}\right)=-d\left(\varepsilon_{1}^{-y} \varepsilon_{2}^{x}\right)$, and the lemma is proved.

Lemma 3: Equation (27) is not satisfied by $(x, y)$ if $x \equiv y \equiv 0(\bmod 2)$.

Proof: We find $\alpha \alpha^{\prime \prime}=\mu^{2} /\left(4 i 2^{\frac{1}{2}}\right)$, where $\mu$ is an integer in $K(\theta)$. Putting $\alpha=\lambda^{2}, \lambda$ being a unit in $K(\theta)$, we obtain $\left(\lambda \lambda^{\prime \prime}\right)^{2}=\mu^{2} /\left(4 i 2^{\frac{1}{2}}\right)$, whence $4 i 2^{\frac{1}{2}}=\mu^{2}\left(\lambda^{\prime} \lambda^{\prime \prime \prime}\right)^{2}$. Since $4 i 2^{\frac{1}{2}}=\left((2+2 i) / 2^{\frac{1}{2}}\right)^{2}$ we conclude that $2^{\frac{1}{4}}$ belongs to $K(\theta)$. It is easily seen that this is impossible.

Lemma 4: Equation (27) is not satisfied by $(x, y)$ if $x \equiv y \equiv 1 \quad(\bmod 2)$.

Proof: Putting $\alpha \varepsilon_{1} \varepsilon_{2}=\lambda^{2}$ we get $\left(\alpha \varepsilon_{1} \varepsilon_{2}\right)\left(\alpha^{\prime \prime} \varepsilon_{1}{ }^{\prime \prime} \varepsilon_{2}{ }^{\prime \prime}\right)=\alpha \alpha^{\prime \prime} \varepsilon_{2}{ }^{2}=$ $\left(\lambda \lambda^{\prime \prime}\right)^{2}$. From the preceding proof it follows that this is impossible.

Lemma 5: The system of equations (26) and (27) is not satisfied by $(x, y)$, either if $x \equiv 0(\bmod 2), y \equiv 1(\bmod 2), t=1$ or if $x \equiv 1(\bmod 2), y \equiv 0$ $(\bmod 2), t=-1$.

Proof: In the first case we find $\alpha \varepsilon_{2}=\mu^{2} /\left(4 i e_{2} 2^{\frac{1}{2}}\right)$ and in the second one $\alpha \varepsilon_{1}=\mu_{1}^{2} /\left(4 i e_{2} 2^{\frac{1}{2}}\right)$. As before we see that these numbers are not squares of any unit in $K(\theta)$.

From these lemmas we conclude that it is sufficient to study the equation

$$
s\left(\varepsilon_{1}^{x} \varepsilon_{2}^{y}\right)+\left(i+2^{\frac{1}{2}}\right) d\left(\varepsilon_{1}^{x} \varepsilon_{2}^{y}\right)= \pm 4, \quad x \text { odd }, y \text { even } .
$$

6. Now we find

$$
\begin{gathered}
\varepsilon_{1}^{8}=1+4 B, \quad B=4 P+\theta Q, \quad P=-7+5 \cdot 2^{\frac{1}{2}}+i\left(11 \cdot 2^{\frac{1}{2}}-14\right), \\
Q=54 \cdot 2^{\frac{1}{2}}-78+i\left(2^{\frac{1}{2}}-4\right),
\end{gathered}
$$




$$
\begin{gathered}
\varepsilon_{2}{ }^{8}=1+4 B_{1}, \quad B_{1}=4 P_{1}+\theta_{1} Q_{1}, \quad P_{1}=-7-5 \cdot 2^{\frac{1}{2}}+i\left(11 \cdot 2^{\frac{1}{2}}+14\right) \\
Q_{1}=-54 \cdot 2^{\frac{1}{2}}-78+i\left(2^{\frac{1}{2}}+4\right) .
\end{gathered}
$$

Putting $x=8 m_{1}+r, y=8 n_{1}+s$, where $r= \pm 1$ or \pm 3 and $s=0$, \pm 2 or 4 , and applying the first lemma of Section 5 , we see that it is sufficient to treat the following eight cases:

$$
\begin{array}{ll}
1^{\circ} r=1, s=0, & 2^{\circ} r=1, s=-2, \\
3^{\circ} r=1, s=2, & 4^{\circ} r=1, s=4, \\
5^{\circ} r=3, s=0, & 6^{\circ} r=3, s=-2, \\
7^{\circ} r=3, s=2, & 8^{\circ} r=3, s=4 .
\end{array}
$$

Let $\beta$ denote any integer in $K(\theta)$. For the sake of brevity we introduce the notation

$$
s\left(\beta \varepsilon_{1}{ }^{r} \varepsilon_{2}^{s}\right)+\left(i+2^{\frac{1}{2}}\right) d\left(\beta \varepsilon_{1}^{r} \varepsilon_{2}^{s}\right)=p\left(\beta \varepsilon_{1}{ }^{r} \varepsilon_{2}{ }^{s}\right) .
$$

Then $p\left(\beta \varepsilon_{1}^{r} \varepsilon_{2}^{s}\right)$ is an integer in $k\left(i 2^{\frac{1}{2}}\right)$. The equation (30) implies

$$
\begin{aligned}
p\left(\varepsilon_{1}{ }^{r} \varepsilon_{2}{ }^{s}\right)+ & 4\left(\begin{array}{c}
m_{1} \\
1
\end{array}\right) p\left(B \varepsilon_{1}{ }^{r} \varepsilon_{2}{ }^{s}\right)+4\left(\begin{array}{c}
n_{1} \\
1
\end{array}\right) p\left(B_{1} \varepsilon_{1}{ }^{r} \varepsilon_{2}{ }^{s}\right)+\ldots \\
& +4^{q} \sum_{k=0}^{q}\left(\begin{array}{c}
m_{1} \\
q-k
\end{array}\right)\left(\begin{array}{c}
n_{1} \\
k
\end{array}\right) p\left(B^{q-k} B_{1}{ }^{k} \varepsilon_{1}{ }^{r} \varepsilon_{2}{ }^{s}\right)+\ldots= \pm 4 .
\end{aligned}
$$

Now we have that $\varepsilon_{1}{ }^{2}, \varepsilon_{1} \varepsilon_{2}$ and $\varepsilon_{2}{ }^{2}$ all belong to the ring

$$
R\left(1,2^{\frac{1}{2}}, i, i 2^{\frac{1}{2}}, \theta, \theta 2^{\frac{1}{2}}, \theta i, \theta i 2^{\frac{1}{2}}\right) .
$$

Hence it is obvious that $p\left(B^{q-k} B_{1}{ }^{k} \varepsilon_{1}{ }^{r} \varepsilon_{2}{ }^{s}\right) \equiv 0 \quad(\bmod 2)$.

The cases $3^{\circ}, 4^{\circ}, 6^{\circ}, 7^{\circ}$ and $8^{\circ}$ can be excluded at once. In fact, we find $p\left(\varepsilon_{1}{ }^{r} \varepsilon_{2}{ }^{s}\right)=-12-16 i 2^{\frac{1}{2}},-140,-44,4+48 i 2^{\frac{1}{2}}$ and $340+96 i 2^{\frac{1}{2}}$, respectively, and further $p\left(B \varepsilon_{1}{ }^{r} \varepsilon_{2}{ }^{s}\right) \equiv p\left(B_{1} \varepsilon_{1}{ }^{r} \varepsilon_{2}{ }^{s}\right) \equiv 0(\bmod 8)$ in all these cases, which contradicts the validity of $(31) \bmod 32$. The remaining three cases must be studied separately.

$2^{\circ}$ : We get $p\left(\varepsilon_{1} \varepsilon_{2}{ }^{-2}\right)=4, p\left(B \varepsilon_{1} \varepsilon_{2}{ }^{-2}\right)=8 \cdot 223-8 \cdot 17 i 2^{\frac{1}{2}}, p\left(B_{1} \varepsilon_{1} \varepsilon_{2}^{-2}\right)=$ $-32 \cdot 3+15 \cdot 8 i 2^{\frac{1}{2}}$ and $p\left(B^{2} \varepsilon_{1} \varepsilon_{2}{ }^{-2}\right) \equiv p\left(B_{1}{ }^{2} \varepsilon_{1} \varepsilon_{2}{ }^{-2}\right) \equiv p\left(B B_{1} \varepsilon_{1} \varepsilon_{2}{ }^{-2}\right) \equiv 0$ $(\bmod 8)$.

Using that $B=\theta 2^{\frac{1}{2}}\left(i+2^{\frac{1}{2}}+2 N\right), B_{1}=\theta_{1} 2^{\frac{1}{2}}\left(i+2^{\frac{1}{2}}-2 N^{\prime \prime}\right)$, where $N$ belongs to $R$, we find

$$
p\left(B^{q-k} B_{1}^{k} \varepsilon_{1}^{r} \varepsilon_{2}^{s}\right)=2^{[q / 2]+1}\left(a_{q k}+b_{q k} i 2^{\frac{1}{2}}\right),
$$

$a_{q k}$ and $b_{q k}$ denoting integers in $k(1)$.

On the right-hand side of $(31)$ we must have +4 . Otherwise (31) could not be valid mod 16. Dividing by 32 we then obtain 


$$
\begin{aligned}
& m_{1}\left(223-17 i 2^{\frac{1}{2}}\right)+n_{1}\left(-12+15 i 2^{\frac{1}{2}}\right)+2\left(f\left(m_{1}, n_{1}\right)+g\left(m_{1}, n_{1}\right) i 2^{\frac{1}{2}}\right) \\
& \quad+2^{3} \sum_{k=0}^{3}\left(\begin{array}{c}
m_{1} \\
3-k
\end{array}\right)\left(\begin{array}{c}
n_{1} \\
k
\end{array}\right)\left(a_{3 k}+b_{3 k} i 2^{\frac{1}{2}}\right)+\ldots \\
& \quad+2^{2 q+[q / 2]-4} \sum_{k=0}^{q}\left(\begin{array}{c}
m_{1} \\
q-k
\end{array}\right)\left(\begin{array}{c}
n_{1} \\
k
\end{array}\right)\left(a_{q k}+b_{q k} i 2^{\frac{1}{2}}\right)+\ldots=0,
\end{aligned}
$$

where $f\left(m_{1}, n_{1}\right)$ and $g\left(m_{1}, n_{1}\right)$ are polynomials in $m_{1}$ and $n_{1}$ with coefficients which are integers in $k(1)$.

The exponent of the highest power of 2 which divides $(q-k) ! k !$ is $\leqq q-1$. The general term in (33) can thus be written in the form

$$
2^{q+[q / 2]-3}\left(f_{q}\left(m_{1}, n_{1}\right)+g_{q}\left(m_{1}, n_{1}\right) i 2^{\frac{1}{2}}\right),
$$

where $f_{q}\left(m_{1}, n_{1}\right)$ and $g_{q}\left(m_{1}, n_{1}\right)$ are polynomials in $m_{1}$ and $n_{1}$ with coefficients which are integers in relation to 2 in $k(1)$.

Now (33) yields the following 2-adic developments:

$$
\begin{aligned}
& 0=m_{1} \quad+2(\quad)+2^{2}(\quad)+2^{3}(\quad)+\ldots, \\
& 0=m_{1}+n_{1}+2()+2^{2}(\quad)+2^{3}(\quad)+\ldots .
\end{aligned}
$$

According to a theorem of Th. Skolem [13, p. 180], the equations (34) have at most one solution $m_{1}, n_{1}$, because

$$
\left|\begin{array}{ll}
1 & 0 \\
1 & 1
\end{array}\right|=1
$$

Obviously this solution is $m_{1}=n_{1}=0$, corresponding to $\alpha=\varepsilon_{1} \varepsilon_{2}{ }^{-2}$. On account of Lemmas 1 and 2 the three other possibilities are $\varepsilon_{1}{ }^{-1} \varepsilon_{2}{ }^{2}, \varepsilon_{1}{ }^{-2} \varepsilon_{2}{ }^{-1}$ and

$$
\varepsilon_{1}^{2} \varepsilon_{2}=\frac{\left(\left(1-i E^{\prime} 2^{\frac{1}{2}}\right) E^{\frac{1}{2}}+\left(1+i 2^{\frac{1}{2}}\right)^{\frac{1}{2}}\right)^{2}}{-2^{\frac{1}{2}}\left(E^{\prime}-i\right)},
$$

the two last units giving $u= \pm 1, v=0, e=e_{2}=1$ and $n=1$. See (22) and (24).

$5^{\circ}:$ Here we find $p\left(\varepsilon_{1}^{3}\right)=4, p\left(B \varepsilon_{1}^{3}\right)=-2^{3} \cdot 21+2^{4} \cdot 165 i 2^{\frac{1}{2}}$,

$$
p\left(B_{1} \varepsilon_{1}^{3}\right)=-2^{4} \cdot 33-2^{4} \cdot 129 i 2^{\frac{1}{2}}
$$

and $p\left(B^{2} \varepsilon_{1}{ }^{3}\right) \equiv p\left(B B_{1} \varepsilon_{1}{ }^{3}\right) \equiv p\left(B_{1}{ }^{2} \varepsilon_{1}{ }^{3}\right) \equiv 0 \quad(\bmod 8)$. As in the previous case we get the 2 -adic developments:

$$
\begin{aligned}
& 0=m_{1} \quad+2(\quad)+2^{2}()+2^{3}(\quad)+\ldots, \\
& 0=m_{1}+n_{1}+2(\quad)+2^{2}()+2^{3}(\quad)+\ldots .
\end{aligned}
$$

The only solution $m_{1}=n_{1}=0$ gives $\alpha=\varepsilon_{1}{ }^{3}, \varepsilon_{1}{ }^{-3}, \varepsilon_{2}{ }^{3}$ or $\varepsilon_{2}{ }^{-3}$; and the first 
two units yield $u=v=1, e=1, e_{2}=-1, n=-1$ and $u=v=-1$ with the same values of $e, e_{2}$ and $n$.

$1^{\circ}$ : If we proceed as in the two previous cases we find that there are at most two solutions $m_{1}, n_{1}$. Since only one solution is known, namely $m_{1}=n_{1}=0$, we have to use other $p$-adic developments in order to prove that no other solutions exist. At first we prove that $m_{1} \equiv n_{1} \equiv 0 \quad(\bmod 8)$. We get

$$
\begin{gathered}
p\left(\varepsilon_{1}\right)=4, \quad p\left(B \varepsilon_{1}\right)=-2^{3 \cdot 69}+2^{4} \cdot 15 i 2^{\frac{1}{2}}, \\
p\left(B_{1} \varepsilon_{1}\right)=2^{6} \cdot 9+2^{5} \cdot 21 i 2^{\frac{1}{2}}, \quad p\left(B^{2} \varepsilon_{1}\right)=-2^{3} \cdot 909-2^{4} \cdot 7785 i 2^{\frac{1}{2}}, \\
p\left(B B_{1} \varepsilon_{1}\right)=-2^{3} \cdot 1355+2^{3} \cdot 679 i 2^{\frac{1}{2}}, \\
p\left(B_{1}{ }^{2} \varepsilon_{1}\right)=-2^{3} \cdot 36297+2^{6} \cdot 339 i 2^{\frac{1}{2}} .
\end{gathered}
$$

Further we find that $p\left(B^{q-k} B_{1}{ }^{k} \varepsilon_{1}\right) \equiv 0 \quad(\bmod 16)$ for $q=3$ and $k=1,2,3$ and for $q=4$ and $k=1,2,3,4$.

As in case $2^{\circ}$ we obtain the equation

$$
\begin{array}{r}
+2^{2}\left\{\left(\begin{array}{c}
m_{1} \\
2
\end{array}\right)\left(-909-2 \cdot 7785 i 2^{\frac{1}{2}}\right)+m_{1} n_{1}\left(-1355+679 i 2^{\frac{1}{2}}\right)\right. \\
\left.+\left(\begin{array}{c}
n_{1} \\
2
\end{array}\right)\left(-36297+2^{3} \cdot 339 i 2^{\frac{1}{2}}\right)\right\} \\
+2^{5} \sum_{k=0}^{3}\left(\begin{array}{c}
m_{1} \\
3-k
\end{array}\right)\left(\begin{array}{c}
n_{1} \\
k
\end{array}\right)\left(a_{k}+b_{k} i 2^{\frac{1}{2}}\right)+2^{7} \sum_{k=0}^{4}\left(\begin{array}{c}
m_{1} \\
4-k
\end{array}\right)\left(\begin{array}{c}
n_{1} \\
k
\end{array}\right)\left(c_{k}+d_{k} i 2^{\frac{1}{2}}\right)+\ldots \\
+2^{2 q+[q / 2]-4} \sum_{k=0}^{q}\left(\begin{array}{c}
m_{1} \\
q-k
\end{array}\right)\left(\begin{array}{c}
n_{1} \\
k
\end{array}\right)\left(a_{q k}+b_{q k} i 2^{\frac{1}{2}}\right)+\ldots=0,
\end{array}
$$

$a_{k}, b_{k}, c_{k}$, and $d_{k}$ being integers in $k(1)$. From (35) it is easily seen that $m_{1} \equiv n_{1} \equiv 0 \quad(\bmod 2)$. Neglecting the trivial solution $m_{1}=n_{1}=0$, we can put $m_{1}=2^{w} m_{2}$ and $n_{1}=2^{w} n_{2}, w \geqq 1$ and $\left(m_{2}, n_{2}\right)=1$. For $q \geqq 5$ the general term in (35) is divisible by $2^{q+[q / 2]+w-2}$, that is at least by $2^{w+5}$. Then it is obvious that $m_{2}$ is even and $n_{2}$ is odd. Now we get the congruence

$$
\begin{gathered}
m_{2}\left(-69+30 i 2^{\frac{1}{2}}\right)+n_{2}\left(72+84 i 2^{\frac{1}{2}}\right)+2 m_{2}\left(2^{w} m_{2}-1\right)\left(-909-2 \cdot 7785 i 2^{\frac{1}{1}}\right) \\
+2^{w+2} m_{2} n_{2}\left(-1355+679 i 2^{\frac{1}{2}}\right)+2 n_{2}\left(2^{w} n_{2}-1\right)\left(-36297+2^{3} \cdot 339 i 2^{\frac{1}{2}}\right) \\
\equiv 0 \quad(\bmod 32) .
\end{gathered}
$$

This gives the following two congruences $\bmod 16$ :

$$
\begin{aligned}
-69 m_{2}+72 n_{2}+1818 m_{2}-2^{w+1}+2 n_{2} \equiv 0 & (\bmod 16), \\
15 m_{2}+42 n_{2}+2 m_{2}-2^{w+1} m_{2} n_{2}-8 \equiv 0 & (\bmod 16) .
\end{aligned}
$$


Simplifying we obtain

$$
\begin{aligned}
5 m_{2}+10 n_{2} & \equiv 2^{w+1} \quad(\bmod 16), \\
m_{2}+10 n_{2} & \equiv 2^{w+1} m_{2} n_{2}+8 \quad(\bmod 16) .
\end{aligned}
$$

Hence $40 n_{2} \equiv 2^{w+1}\left(5 m_{2} n_{2}-1\right)+8(\bmod 16)$, and thus $w \geqq 3$. Now we find $\varepsilon_{1}{ }^{64} \equiv 1\left(\bmod \left(11-6 i 2^{\frac{1}{2}}\right)\right)$ and $\varepsilon_{1}{ }^{192} \equiv 1 \quad(\bmod 193)$. In the next section we will use 193-adic developments in order to prove that $m_{1}=n_{1}=0$ is the only solution of $(31)$ in case $1^{\circ}$.

7. Cumbersome calculations give us

$$
\begin{aligned}
& \varepsilon_{1}{ }^{16}=-174015+122176 \cdot 2^{\frac{1}{2}}+i\left(212096-149824 \cdot 2^{\frac{1}{2}}\right) \\
& \quad+\theta\left\{\left(128400-90448 \cdot 2^{\frac{1}{2}}\right)+i\left(296672-210040 \cdot 2^{\frac{1}{2}}\right)\right\}, \\
& \varepsilon_{1}{ }^{32} \equiv-16019+7437 \cdot 2^{\frac{1}{2}}+i\left(5320-11580 \cdot 2^{\frac{1}{2}}\right) \\
& \quad+\theta\left\{\left(2319+11264 \cdot 2^{\frac{1}{2}}\right)+i\left(14153+17228 \cdot 2^{\frac{1}{2}}\right)\right\} \quad\left(\bmod 193^{2}\right), \\
& \varepsilon_{1}{ }^{96} \equiv-17537 \cdot 2^{\frac{1}{2}}+193 \theta\left\{\left(-11+44 \cdot 2^{\frac{1}{2}}\right)+i\left(-86-86 \cdot 2^{\frac{1}{2}}\right)\right\} \quad\left(\bmod 193^{2}\right), \\
& \varepsilon_{1}{ }^{192} \equiv 1+193 \theta\left\{\left(7-56 \cdot 2^{\frac{1}{2}}\right)+i\left(-66-33 \cdot 2^{\frac{1}{2}}\right)\right\} \quad\left(\bmod 193^{2}\right) .
\end{aligned}
$$

We have $\varepsilon_{1}{ }^{192}=1+193 C$ and $\varepsilon_{2}{ }^{193}=1+193 C_{1}$, where

$$
\begin{aligned}
C & \equiv\left(7-56 \cdot 2^{\frac{1}{2}}\right)+i\left(-66-33 \cdot 2^{\frac{1}{2}}\right) \quad(\bmod 193), \\
C_{1} & \equiv\left(7+56 \cdot 2^{\frac{1}{2}}\right)-i\left(-66+33 \cdot 2^{\frac{1}{2}}\right) \quad(\bmod 193) .
\end{aligned}
$$

If in (30) we insert $x=192 m_{3}+64 r_{1}+1$ and $y=192 n_{3}+64 s_{1}$ we get the 193-adic development

$$
p\left(\varepsilon_{1}{ }^{64 r_{1}+1} \varepsilon_{2}{ }^{64 s_{1}}\right)+193()+193^{2}()+193^{3}()+\ldots=4 .
$$

Here is $r_{1}=-1,0$ or 1 and $s_{1}=-1,0$ or 1 . The first condition to be fulfilled is

$$
p\left(\varepsilon_{1}{ }^{64 r_{1}+1} \varepsilon_{2}{ }^{64 s_{1}}\right) \equiv 4 \quad(\bmod 193) .
$$

This implies $r_{1}=s_{1}=0$. In the remaining eight cases we find, in fact, denoting for brevity the left-hand side of the congruence (37) by $\left(r_{1}, s_{1}\right)$ :

$$
\begin{gathered}
(0,-1) \equiv 60-13 i 2^{\frac{1}{2}} ; \quad(0,1) \equiv-58-89 i 2^{\frac{1}{2}}, \quad(1,0) \equiv 49-7 i 2^{\frac{1}{2}} \\
(1,1) \equiv 33-86 i 2^{\frac{1}{2}}, \quad(1,-1) \equiv 65+72 i 2^{\frac{1}{2}} ; \quad(-1,0) \equiv-47-95 i 2^{\frac{1}{2}}, \\
(-1,1) \equiv 83-8 i 2^{\frac{1}{2}}, \quad(-1,-1) \equiv 22-80 i 2^{\frac{1}{2}},
\end{gathered}
$$

the congruences being mod 193. In the calculations we make use of the fact that

$$
\varepsilon_{1}{ }^{64} \equiv-48-61 i 2^{\frac{1}{2}}+\theta\left\{\left(27-78 \cdot 2^{\frac{1}{2}}\right)+i\left(50+73 \cdot 2^{\frac{1}{2}}\right)\right\} \quad(\bmod 193) .
$$


The equation (36) can now be written

$$
m_{3} p\left(C \varepsilon_{1}\right)+n_{3} p\left(C_{1} \varepsilon_{1}\right)+193(\quad)+193^{2}(\quad)+\ldots=0 .
$$

Further we find $p\left(C \varepsilon_{1}\right) \equiv 88-14 i 2^{\frac{1}{2}}(\bmod 193)$ and $p\left(C_{1} \varepsilon_{1}\right) \equiv$ $80+60 i 2^{\frac{1}{2}} \quad(\bmod 193)$, and hence

Since

$$
\begin{aligned}
& 0=88 m_{3}+80 n_{3}+193(\quad)+193^{2}(\quad)+\ldots, \\
& 0=-14 m_{3}+60 n_{3}+193()+193^{2}(\quad)+\ldots
\end{aligned}
$$

$$
\left|\begin{array}{cc}
88 & 80 \\
-14 & 60
\end{array}\right| \neq 0 \quad(\bmod 193)
$$

the only solution is $m_{3}=n_{3}=0$, according to the theorem of Th. Skolem mentioned in Section 6. Hence $x=1, y=0$, that is, $\alpha=\varepsilon_{1}, \varepsilon_{1}^{-1}, \varepsilon_{2}$ or $\varepsilon_{2}{ }^{-1}$. To $\varepsilon_{1}$ corresponds the solution $u=1, v=0, e=-1, e_{2}=1$ and $n=0$; to $\varepsilon_{1}{ }^{-1}$ corresponds the solution $u=-1, v=0$ with the same values of $e, e_{2}$ and $n$.

Then it is shown that the only solutions of $u$ and $v$ are $u= \pm 1, v=0$; $u=1, v=1 ; u=-1, v=-1$. Hence our theorem in Section 1 is proved.

\section{REFERENCES}

1. B. Delaunay, On the complete solution of the equation $X^{3} \varrho+Y^{3}=1$, Publ. Soc. Math. Charkow (1916). (Russian.) See also his paper: Vollständige Lösung der unbestimmten Gleichung $X^{3} q+Y^{3}=1$ in ganzen Zahlen, Math. Z. 28 (1928), 1-9.

2. P. Häggmark, On an unsolved question concerning the diophantine equation $A x^{3}+B y^{3}=C$, Ark. Mat. 1 (1950), 279-294.

3. W. Ljunggren, Einige Eigenschaften der Einheiten reeller quadratischer und rein-biquadratischer Zahlkörper usw., Skr. Norske Vid. Akad. Oslo, Mat.-Naturv. Klasse, 1936 No. 12, 1-73.

4. W. Ljunggren, Zur Theorie der Gleichung $x^{2}+1=D y^{4}$, Avh. Norske Vid. Akad. Oslo, Mat.-Naturv. Klasse, 1942 No. 5, 1-27.

5. T. Nagell, Vollständige Lösung einiger unbestimmten Gleichungen dritten Grades, Skr. Norske Vid. Akad. Oslo, Mat.-Naturv. Klasse, 1922 No. 14, 1-13.

6. T. Nagell, Uber die Einheiten in reinen kubischen Zahlkörpern, Skr. Norske Vid. Akad. Oslo, Mat.-Naturv. Klasse, 1923 No. 11, 1-34.

7. T. Nagell, Solution complète de quelques équations cubiques à deux indéterminées, J. Math. pur. appl., (9) 4 (1925), 209-270.

8. T. Nagell, Einige Gleichungen von der Form $a y^{2}+b y+c=d x^{3}$, Avh. Norske Vid. Akad. Oslo, Mat.-Naturv. Klasse, 1930 No. 7, 1-15.

9. T. Nagell, Zahlentheoretische Notizen VII-IX, Norsk mat. forenings skrifter, Serie 1 No. 17 (1927), 1-23.

10. T. Nagell, Zahlentheoretische Sätze, Avh. Norske Vid. Akad. Oslo, Mat.-Naturv. Klasse, 1930 No. 5, 1-12.

11. C. L. Siegel, Über einige Anwendungen diophantischer Approximationen, Abh. preuss. Akad. Wiss., Phys.-math. Kl., 1929 Nr. 1, 1-70. 
12. Th. Skolem, Einige Sätze über gewisse Reihenentwicklungen und exponentiale Beziehungen mit Anwendung auf diophantische Gleichungen, Skr. Norske Vid. Akad. Oslo, Mat.-Naturv. Klasse, 1933 No. 6, 1-61.

13. Th. Skolem, Ein Verfahren zur Behandlung gewisser exponentialer Gleichungen und diophantischer Gleichungen, $8^{\text {de }}$ Skandinaviska Matematikerkongressen i Stockholm 1934, 163-188.

14. Th. Skolem, Einige Sätze über p-adische Potenzreihen mit Anwendung auf gewisse exponentielle Gleichungen, Math. Ann. 111 (1935), 399-424.

15. Th. Skolem, Anwendung exponentieller Kongruenzen zum Beweis der Unlösbarkeit gewisser diophantischer Gleichungen, Avh. Norske Vid. Akad. Oslo, Mat.-Naturv. Klasse, 1937 No. 12, 1-16.

UNIVERSITY OF BERGEN, NORWAY. 